Artículo Original. Enero-Diciembre 2019; 9:1-12. Recibido: 08/03/2019 Aceptado: 04/07/2019.

http://dx.doi.org/10.21929/abavet2019.912

\title{
Producción de gas en heces de bovinos adicionando taninos directos o sobre la dieta
}

Gas production in bovine feces adding tannins directly or on the diet

\author{
Murillo-Ayala Eva1'iD xitla.muri@gmail.com Corona-Palazuelos Melissa ${ }^{1}$ (iD \\ corona_melissa@hotmail.com Velázquez-Elenes Ernesto1 \\ mvzernesto_vquez@hotmail.com Rubén Alberto Davicino2@ic \\ rdavicino@ayv.unrc.edu.ar Romo-Rubio Javier110 romo60@uas.edu.mx

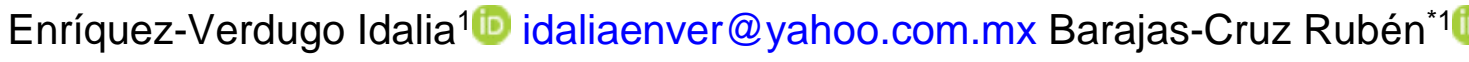 \\ rubar@uas.edu.mx
}

\begin{abstract}
${ }^{1}$ Facultad de Medicina Veterinaria y Zootecnia, Universidad Autónoma de Sinaloa. Culiacán, México. ${ }^{2}$ Facultad de Agronomía y Veterinaria, Universidad Nacional del Río Cuarto, Córdoba, Argentina. *Autor responsable y de correspondencia: Barajas-Cruz Rubén. Facultad de Medicina Veterinaria y Zootecnia de la Universidad Autónoma de Sinaloa. Boulevard San Ángel s/n. Colonia San Benito. CP 80246. Culiacán, Sinaloa, México.
\end{abstract}

\section{RESUMEN}

Se realizaron dos experimentos para conocer el efecto de los extractos de taninos (ET) en la producción de gas. En el experimento 1 se colectaron heces de cinco toretes $(420 \pm 10 \mathrm{~kg})$ de un mismo corral durante cuatro días consecutivos para tener una muestra por día, $100 \mathrm{~g}$ de las heces se colocaron en frascos de $600 \mathrm{~mL}$ para cada muestra y se adicionaron los siguientes tratamientos: TE) heces sin tratar y HT) $7.4 \%$ de ET condensados. En el experimento 2 se aplicaron los siguientes tratamientos: TES) dieta sin ET, TC) $0.6 \%$ de taninos condensados y $\mathrm{TH}$ ) $0.6 \%$ de taninos hidrolizables a la dieta de 18 becerros $(220 \pm 9 \mathrm{~kg}$ ) durante 28 días. En los dos experimentos las heces obtenidas se incubaron durante $24 \mathrm{~h}$ para medir su producción de gas por desplazamiento. Los datos obtenidos de gas se compararón con Diseño experimental de bloques al azar para eliminar el efecto día de muestreo. En el primer experimento la producción de gas disminuyó $44 \%(P<0.001)$ con los extractos de taninos adicionados sobre las heces, pero adicionados a través de la dieta no hubo diferencias entre los tratamientos.

Palabras clave: extracto de taninos, heces, producción de gas, bovinos en engorda.

\section{ABSTRACT}

Two experiments were performed to determine the influence of (TE) tannins extract addition on gas production from beef-cattle feces. Experiment 1: fecal samples collected during four consecutive days from five bullocks placed in a pen, and an integrated pull sample/day. $100 \mathrm{~g}$ of feces was placed in $600 \mathrm{ml}$ flasks, in a randomized complete block design into treatments: 1) Feces without TE addition (Control), and 2) Addition Dry Matter Basis (DB) with 7.4\% of condensed TE (CT). In the second experiment, eighteen calves in a complete randomized block design were used treatments were: 1) diet without TE (Control), 2) Control plus $0.6 \%$ DB of CT, and 3 ) Control plus $0.6 \%$ DB of Hydrolyzable TE (HT) on a 28 days diet. Total of feces was incubated for $24 \mathrm{~h}$ in vitro, then gas production was measured, and the results analyzed by ANOVA. CT addition decreased by $44 \%$ gas production $(\mathrm{P}<0.001)$. The TE addition does not modify gas production $(P>0.50)$; results indicate that gas production decreases with the addition of TE on bovine feces, but, does not when TE is included in the diet.

Keywords: tannins extract, feces, gas production, beef-cattle. 


\section{INTRODUCCIÓN}

El manejo de las excretas y el aumento en el tamaño de las instalaciones de bovinos en confinamiento se relaciona cada vez más con el impacto al medioambiente (Adeola, 1999; Kebreab et al., 2009; Cardona-Iglesias, 2016), por consecuencia la reducción de la emisión de sólidos, líquidos y gases son factores que presionan a la ganadería intensiva (Archibeque et al., 2006; Grainger y Beauchemin, 2011). Los gases producidos por la volatilización de las sustancias orgánicas de las heces de los bovinos contribuyen a la diseminación de olores indeseables en el ambiente (Varel y Miller, 2000; Shabtay et al., 2009). Una alternativa para disminuir la cantidad de gases producidos por las heces de los bovinos en engorda, es mediante el uso de taninos (Cardona-Iglesias, 2016; BarrosRodríguez et al., 2017). Los taninos son compuestos polifenólicos, producto del metabolismo secundario de una amplia variedad de plantas (Frutos et al., 2004; Vélezterranova et al., 2014) que se relaciona con la protección contra infección, insectos o animales herbívoros (Duval y Averous, 2016). Los taninos se clasifican en dos grupos: taninos hidrolizables y taninos condensados (Frutos et al., 2004; Vázquez et al., 2012; Kardel et al., 2013). Los extractos de taninos (ET) modifican la fermentación en el rumen y reducen la producción de gas (Bernal et al., 2008; Lascano y Cárdenas, 2010; Cárdenas, 2012). Los ET tienen la capacidad de unirse a las proteínas de la membrana celular de las bacterias, cambian su estructura, función, e inhiben su capacidad de unirse a las partículas de alimento y la fermentación de sus nutrientes (Cárdenas, 2012; BarrosRodríguez et al., 2017). Con base en lo anterior se planteó la hipótesis que la adición de extracto de taninos tanto condensados como hidrolizables disminuye la cantidad de gas producida a partir de las heces de los bovinos en engorda. Por lo tanto, el objetivo de la investigación fue determinar la influencia de la producción de gas en heces de bovinos en engorda adicionando taninos directos o sobre la dieta.

\section{MATERIAL Y MÉTODOS}

La presente investigación estuvo constituida por dos experimentos, ambos se realizaron en la Unidad Experimental para Bovinos en Engorda en Trópico Seco localizada en la engorda comercial Ganadera Los Migueles, S.A. de C.V. ( $24^{\circ} 51^{\prime}$ N, y $107^{\circ} 26^{\prime}$ O) y en el laboratorio de Investigación en Nutrición y Producción Animal de la FMVZ de la Universidad Autónoma de Sinaloa, ambos ubicado en Culiacán, Sinaloa. Todos los animales usados en la investigación fueron manejados siguiendo recomendaciones internacionales para el cuidado de los animales utilizados en investigación (FASS, 2010).

Experimento 1. En este experimento se evaluó la influencia de la adición de extracto de taninos directamente a las heces de bovinos en engorda sobre la producción de gas in vitro. Se utilizaron cinco toretes Brahman (420 $\pm 10 \mathrm{~kg}$ ) alojados en un corral con piso de tierra $(6 \times 12 \mathrm{~m})$ con $24 \mathrm{~m}^{2}$ de techo metálico que proporcionó $4.8 \mathrm{~m}^{2}$ de sombra por cada 
torete, $2.4 \mathrm{~m}$ de comedero lineal de concreto y $0.6 \mathrm{~m}$ de bebedero con acceso permanente de agua limpia y fresca para los animales.

Durante 60 días antes de tomar las muestras de las heces, los toretes fueron alimentados a libre acceso con una dieta de finalización que tuvo una proporción 10:90 de forraje:concentrado (13.6\% PC; 2.1 Mcal $E N_{m}$ ), formulada con rastrojo de maíz, pasta de soya y grano de maíz molido (Cuadro 1). Una vez adaptados perfectamente a la dieta, durante cuatro días continuos se tomaron muestras de heces obtenidas del recto de cada uno de los toretes $( \pm 200 \mathrm{~g}$ ), las muestras se mezclaron en una licuadora durante $5 \mathrm{~min}$ para obtener una muestra compuesta para cada uno de los cuatro días de muestreo.

Cuadro 1. Composición de las dietas utilizadas en los experimentos de producción de gas de
\begin{tabular}{lcc} 
heces. & \multicolumn{2}{c}{ Proporción en la dieta, \% en BS } \\
\hline Ingredientes & Experimento 1 & Experimento 2 \\
\hline Ensilado de maíz & - & 46.11 \\
Rastrojo de maíz & 10.24 & 25.33 \\
Maíz molido & 68.57 & - \\
Pasta de soya & 7.17 & 16.28 \\
Melaza de caña & 6.82 & 8.29 \\
Sebo & 4.50 & - \\
Ganamin Total Sinaloa & - & 2.61 \\
Ganamin Los Migueles & 2.70 & - \\
Ganabuffer & - & 1.38 \\
Total & $100 \%$ & $100 \%$
\end{tabular}

Análisis calculado (en base seca) ${ }^{\dagger}$

$\begin{array}{lll}\mathrm{PC}, \% & 13.60 & 15.21 \\ \mathrm{ENm}, \text { Mcal kg}^{-1} & 2.113 & 1.358 \\ \mathrm{ENg}, \text { Mcal kg }^{-1} & 1.438 & 0.793\end{array}$

I Ganamin Total Sinaloa (premezcla de vitaminas y minerales; Técnica Mineral Pecuaria S.A. de C.V.; Guadalajara, Jalisco, México). Contienen 68\% PC como NNP y $25 \mathrm{~g}$ de monensina sódica (Rumensin 200 ${ }^{\circledR}$; ElancoAnimal Health).

$\S$ Ganamin Los Migueles (premezcla de vitaminas y minerales; Técnica Mineral Pecuaria S.A. de C.V.; Guadalajara, Jalisco, México). Contienen $112 \%$ PC a como NNP y $25 \mathrm{~g}$ de monensina sódica (Rumensin $200^{\circledR}$; Elanco Animal Health, Indianapolis, IN, USA).

P Ganabuffer (premezcla de amortiguadores del pH; Técnica Mineral Pecuaria S.A. de C.V.; Guadalajara, Jalisco, México).

† Valores calculados con base a valores publicados NASEM, 2016.

De la muestra compuesta de heces, se tomaron alícuotas de $20 \mathrm{~g}$ para determinar el contenido de materia seca (MS) en estufa de aire forzado a $105^{\circ} \mathrm{C}$ durante $24 \mathrm{~h}$ (AOAC, 1997).

Por separado, $400 \mathrm{~g}$ de heces se dividieron en 2 partes de $200 \mathrm{~g}$, a cada una se le adicionaron $200 \mathrm{~g}$ de $\mathrm{H}_{2} \mathrm{O}$ destilada y de manera aleatoria se asignaron a uno de los dos tratamientos que se describen a continuación: 1) heces solas sin la adición de extracto de taninos (Testigo); y 2) heces adicionadas con $7.4 \%$ de extracto de taninos 
condensados en base hidratada (TC). Los TC fueron proporcionados utilizando Bypro $\AA$ (INDUNOR; Buenos Aires, Argentina) una preparación a base de extracto de taninos condensados del árbol de quebracho rojo con un contenido promedio de $75 \%$ de taninos condensados.

Durante los cuatro días, $100 \mathrm{~g}$ de la mezcla homogenizada de heces con agua de cada uno de los tratamientos se colocaron en frascos de $600 \mathrm{~mL}$ con tapón de rosca acondicionados con un tubo colector de gas (Tygon®; Saint-Gobain; Francia) colocados en baño María a $37^{\circ} \mathrm{C}$. En el extremo opuesto del tubo se colocó una probeta de vidrio graduada de $250 \mathrm{~mL}$ llena con agua destilada colocada en posición invertida dentro de un baño de agua (Miller y Varel 2001; Miller et al., 2006) identificada con los datos del tratamiento respectivo. Las muestras se incubaron durante $24 \mathrm{~h}$ y la producción de gas se expresó en $\mathrm{mL}$ como la cantidad de agua desplazada por el gas dentro de cada probeta (Miller y Varel 2001; Miller et al., 2006).

Experimento 2. En este experimento se evaluó la influencia del consumo de dietas adicionadas con extracto de taninos condensados e hidrolizables en la producción de gas en las heces de bovinos en engorda. Se utilizaron 18 becerros Brahman $(220 \pm 6 \mathrm{~kg})$ recién llegados al corral de engorda; al momento de su arribo al corral de engorda, los becerros fueron identificados con aretes plásticos numerados, pesados individualmente y recibieron bacterinas para prevenir enfermedades causadas por Clostridia, Histophilus somni (Ultrabac-sumnovac; Zoetis) y Mannheimia haemolytica (OneShot; Zoetis). En grupos de tres becerros, los animales fueron alojados en seis corraletas con piso de tierra (6 x $12 \mathrm{~m})$, con $24 \mathrm{~m}^{2}$ de techo metálico que proporciona $8 \mathrm{~m}^{2}$ de sombra por cada becerro, $2.45 \mathrm{~m}$ de comedero lineal de concreto y $0.6 \mathrm{~m}$ de bebedero con acceso permanente de agua limpia y fresca para los animales. Los becerros fueron alimentados en condiciones de libre acceso con una dieta de crecimiento con proporción 70:30 de forraje:concentrado (15\% PC; 1.4 Mcal ENm), formulada con ensilado de maíz, pasta de soya y grano de maíz molido (Cuadro 1). Los animales tuvieron un periodo de adaptación de $21 \mathrm{~d}$ a la dieta, interacción social y manejo experimental. Después de los $21 \mathrm{~d}$ del periodo de adaptación, se tomaron muestras de heces directamente del recto de cada becerro, durante tres días consecutivos. Las muestras de heces se manejaron de forma individual, colocadas en bolsas de plástico, identificadas con el número del becerro y día de muestreo; las heces fueron transportadas inmediatamente al laboratorio, donde se midió la producción de gas in vitro con un procedimiento similar al descrito en el Experimento 1.

Una vez obtenidas las muestras de heces, los becerros fueron asignados aleatoriamente a uno de tres tratamientos: 1) dieta de crecimiento sin ET (Testigo); 2) Testigo más 0.6\% en base seca de extracto de taninos condensados (TC); y 3) testigo más $0.6 \%$ en base seca de extracto de taninos hidrolizables (TH). EI TC fue proporcionado en forma de un 
extracto de taninos condensados del árbol de quebracho (Schinopsis balansae), disponible en el mercado como Bypro ${ }^{\circledR}$ (INDUNOR, S. A.; Buenos Aires, Argentina); y el TH se proporcionó a partir de un extracto de taninos de castaño (Castanea sativa), fue proporcionado como NutiP ${ }^{\circledR}$ (SilvaTeam; San Michele Mondavi, Italia); ambos extractos con un contenido de $75 \%$ de taninos.

La dosis diaria correspondiente de TC y TH de acuerdo al tratamiento asignado por corral, se dispersaron en $1000 \mathrm{~g}$ de maíz molido. Después se mezcló y homogenizó manualmente durante cinco minutos en el interior de una bolsa de plástico transparente (etiquetado con el número del corral y tratamiento); la mezcla de maíz molido con el ET correspondiente se adicionó en el comedero de cada corral al momento de servir el alimento $(11: 00 \mathrm{~h})$, se mezcló manualmente con el alimento contenido en el tercio superior del comedero (procedimiento Top dress). En los corrales asignados al tratamiento testigo se les ofreció diariamente $1000 \mathrm{~g}$ de maíz molido para igualar el manejo de la alimentación y la ingesta de energía con el resto de los animales involucrados en el experimento. Los becerros fueron alimentados durante $28 \mathrm{~d}$ con sus respectivos tratamientos; una vez completado este periodo, nuevamente se tomaron muestras de heces de cada becerro durante tres días consecutivos con el procedimiento descrito anteriormente. Las heces se colocaron en bolsas de plástico, cerradas, identificadas y transportadas al Laboratorio para medir la producción de gas in vitro como en el procedimiento antes descrito en el Experimento 1; constituyendo cada día una corrida independiente, que a su vez conformó un bloque y los frascos fermentados con las heces de cada animal en cada corrida constituyeron la unidad experimental.

\section{Análisis estadístico}

Los datos de desplazamiento obtenidos en los dos experimentos se analizaron con un diseño estadístico de bloques al azar consideran el día de muestreo como bloque (Hicks, 1973). Para los cálculos estadísticos se usó el programa Statistix ${ }^{\circledR}$ versión 9 (Analytical Software, 2007) y el modelo matemático fue:

$$
Y_{i j k}=\mu+\beta_{i}+\tau_{j}+\varepsilon_{i j k}
$$

\section{RESULTADOS Y DISCUSIÓN}

Experimento 1. Los resultados de la influencia de la adición de extracto de taninos a las heces de bovinos en la producción de gas in vitro se presentan en el Cuadro 2. La adición de $7.4 \%$ de TC (BS) a las heces de bovinos redujo en $45 \%(P<0.001)$ la producción de gas in vitro. 
De acuerdo con la hipótesis planteada, la disminución en la cantidad de gas producida en las heces de los bovinos a las que les fueron adicionados TC, es una respuesta esperada, considerando que las bacterias degradan los componentes de la materia orgánica, y por consecuencia de su metabolismo son productoras de gas (Dhanoa et al., 2004). Cuando éstos microorganismos están en contacto con los taninos condensados se unen a las proteínas de la membrana celular de las bacterias, inhiben su capacidad de ligarse a los sustratos, reducen la fermentación de los nutrientes y la producción de gas (Bae et al., 1993; Jones et al., 1994; Cárdenas, 2012).

Cuadro 2. Influencia de la adición de extracto de taninos a las heces de bovinos en la producción de gas in vitro (Experimento 1).

\begin{tabular}{|c|c|c|c|c|}
\hline \multirow[t]{2}{*}{ Variables } & \multicolumn{2}{|c|}{ Tratamientos } & \multirow[t]{2}{*}{$\mathrm{EEM}^{+}$} & \multirow[t]{2}{*}{$P<$} \\
\hline & Testigo & Taninos & & \\
\hline \multicolumn{5}{|l|}{ Extracto de taninos, $\mathrm{g}^{\S}$} \\
\hline Gramos ET muestra $^{-1}$ & 0.00 & 1.003 & & \\
\hline$\%$ de las heces, $\mathrm{BH}$ & 0.00 & 2.00 & & \\
\hline$\%$ de las heces, BS & 0.00 & 7.41 & & \\
\hline MS de las heces ${ }^{\S}$ & 26.90 & 26.90 & 0.532 & 1.00 \\
\hline \multicolumn{5}{|l|}{ Muestra de heces $\S$} \\
\hline $\mathrm{g}$ (por frasco BH) & 50.20 & 50.29 & 0.037 & 0.12 \\
\hline $\mathrm{g}$ (por frasco BS) & 13.50 & 13.53 & 0.010 & 0.12 \\
\hline \multicolumn{5}{|l|}{ Producción de gas $\$$} \\
\hline Total, en $24 \mathrm{~h}, \mathrm{~mL}$ & 218.33 & 121.33 & 7.636 & $<0.001$ \\
\hline Por $\mathrm{g}$ de heces $\mathrm{BH}, \mathrm{mL}$ & 4.35 & 2.41 & 0.153 & $<0.001$ \\
\hline Por $\mathrm{g}$ de heces $\mathrm{BS}, \mathrm{mL}$ & 16.24 & 8.92 & 0.603 & $<0.001$ \\
\hline
\end{tabular}

${ }^{\dagger}$ Error estándar de la media

$\S$ Valores promedio de 12 observaciones.

Dhanoa et al. (2004) probaron la utilidad de usar heces de bovino como inóculo en lugar de emplear líquido ruminal para desarrollar la prueba de fermentación in vitro; por lo que es posible inferir que la tendencia en los procesos de fermentación es similar con ambos tipos de inóculos, aunque la magnitud de la respuesta es más baja cuando se utilizan heces dada la menor cantidad de células microbianas por gramo de inóculo. Con base en lo anterior, el comportamiento fermentativo de las bacterias presentes en las heces como resultado de la adición de ET, es explicable en los mismos términos que cuando este fenómeno se ha apreciado utilizando como inóculo líquido ruminal.

En una serie de estudios in vitro se atribuyó una disminución en la producción de gas por parte de los microbios del rumen a la presencia de taninos en varias especies de leguminosas tropicales (Carmona et al., 2005; Bernal et al., 2008; Cárdenas, 2012). De igual manera, la adición de diferentes tipos de TC provenientes de Leucaena (Leucaena leucocephala) disminuyeron la producción de gas in vitro en relación directa con su peso 
molecular cuando se utilizaron microbios ruminales como inóculo (Huang et al., 2011); por lo que en el presente experimento, la menor producción de gas en las heces a las que se les adicionaron los TC, es presumible que se debe a la acción inhibitoria de los taninos en la actividad microbiana debido al daño estructural que produce en la membrana celular, especialmente en las bacterias Gram-negativas (Bae et al., 1993; Jones et al., 1994). Otros investigadores Gilroyed et al. (2015) apreciaron una disminución en la población de bacterias y protozoarios, así como en la producción de gas de heces de bovinos cuando se adicionaron a la dieta $2.5 \%$ de taninos condensados de acacia negra (Acacia mearnsii).

Experimento 2. Los resultados de la influencia de la adición de extracto de taninos a la dieta en la producción de gas de las heces de becerros se presentan en el Cuadro 3. La adición de $0.6 \%$ de ET a la dieta no modificó la producción de gas en las heces. Estos resultados indican que cuando los TC de quebracho o los TH de castaño se adicionan a la dieta en proporciones menores al 1\%, no inducen cambio alguno en la cantidad de gas producido a partir de las heces, a diferencia de lo que se encontró en el Experimento 1, en el cual la adición de $7.4 \%$ de TC (BS) directamente a las heces después de ser excretadas, disminuyó la producción de gas.

Una probable explicación del resultado, es que los taninos a su paso por el aparato digestivo de los bovinos, pueden presentar interacciones; primero con los componentes iniciales de la dieta y después con los productos de la digestión, ya que los taninos tienen dependencia del pH para expresar o no su capacidad para enlazar proteínas de manera estable (Jones et al., 1994). Cuando los taninos son ingeridos, en el rumen se forman complejos tanino-proteína estables el intervalo de pH 3.5 a 7.0 (Makkar, 2003; Beserra et al., 2011), pero se disocian en abomaso y duodeno, donde el pH en bovinos de engorda es generalmente <3.0 (Frutos et al., 2004; Jones et al., 1994).

Cuadro 3. Influencia de la adición de extracto de tanino a la dieta en la producción de gas de las heces de becerros (Experimento 2).

\begin{tabular}{|c|c|c|c|c|c|}
\hline \multirow[t]{2}{*}{ Variables } & \multicolumn{3}{|c|}{ Tratamientos $^{\dagger}$} & \multirow[t]{2}{*}{ EEM } & \multirow[t]{2}{*}{$P<$} \\
\hline & Testigo & $\mathrm{TC}^{\dagger}$ & $\mathrm{TH}^{\dagger}$ & & \\
\hline \multicolumn{6}{|l|}{ Producción de gas, $\mathrm{mL}^{\S}$} \\
\hline Producción previa & 36.0 & 40.9 & 40.1 & 3.681 & 0.57 \\
\hline Producción posterior & 48.5 & 56.1 & 49.1 & 4.854 & 0.43 \\
\hline Diferencia entre periodos & 12.5 & 15.2 & 9.0 & 6.360 & 0.78 \\
\hline \multicolumn{6}{|c|}{ 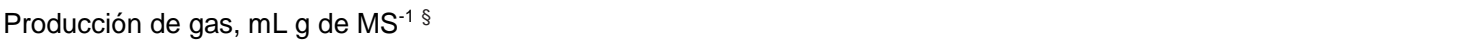 } \\
\hline Producción previa & 4.36 & 5.07 & 4.80 & 0.447 & 0.53 \\
\hline Producción posterior & 5.60 & 6.36 & 5.85 & 0.486 & 0.54 \\
\hline Diferencia entre periodos & 1.25 & 1.29 & 1.05 & 0.672 & 0.97 \\
\hline
\end{tabular}

${ }^{\dagger} \mathrm{TC}=$ Extracto de taninos condensados; $\mathrm{TH}=$ Extracto de taninos hidrolizables

"Error estándar de la media.

$\S$ Valores promedio de 18 observaciones. 
A medida que el quimo avanza en el intestino delgado, el $\mathrm{pH}$ se eleva gradualmente debido a varias secreciones (Owens et al., 1986; Brake et al., 2014), en especial las provenientes del páncreas con concentraciones altas de iones bicarbonato (Huntington et al., 2016). En medios en los que los valores de $\mathrm{pH}$ oscilan de ligeramente alcalinos a ligeramente ácidos como los que prevalecen en el íleon e intestino grueso (Owens et al., 1986; Brake et al., 2014), es factible que los taninos formen de nuevo complejos con componentes no digeridos de la dieta, y con proteínas endógenas de las secreciones digestivas (Frutos et al., 2004; Bae et al., 1993). De esta manera, al momento en que los taninos en la cantidad utilizada en este experimento arribaron al intestino grueso, probablemente ya se encontraban asociados a otros sustratos y por lo tanto, no estaban disponibles para formar nuevos complejos con las proteínas de la membrana celular de las bacterias presentes en este órgano, en consecuencia no modificaron su actividad fermentativa. En ese mismo sentido, al ser excretado el bolo fecal, los taninos se encontraron previamente ligados a otras sustancias e imposibilitados para unirse con las proteínas de las bacterias fecales, por lo que bajo estas circunstancias los taninos no son capaces de modificar la producción de gas en las heces de los bovinos.

Otra posibilidad, es que el nivel de inclusión de los taninos en la dieta de $0.6 \%$ BS del extracto en el experimento actual, haya sido insuficiente para permitir mostrar su actividad, comparado con la cantidad cuatro veces mayor de TC de acacia negra (Acacia mearnsii) que Gilroyed et al. (2015) utilizaron en un experimento de metabolismo ruminal en el que encontraron una disminución en la producción de gas en las heces cuando adicionaron $2.5 \%$ de TC de acacia. Sin embargo, los autores (Gilroyed et al., 2015) no describen el impacto de ese nivel de taninos en el consumo de materia seca y la digestibilidad de la dieta en los bovinos que emplearon.

En el actual experimento, se limitó la cantidad máxima de taninos a ofrecer a un nivel en el que no se viera afectado el consumo de alimento ( $0.6 \%)$, dado que los becerros se encontraban bajo condiciones de alimentación similares a las que se utilizan en la industria con el propósito que los resultados pudieran tener aplicación práctica en la producción de carne de bovinos. De manera general, se considera el nivel de 1.5\% como el umbral máximo de taninos presentes en la dieta de los rumiantes y que cuando se proporcionan taninos hidrolizables en concentraciones mayores los metabolitos productos de la hidrolisis de los taninos en el rumen pueden ser absorbidos a la sangre y afectar negativamente a otros órganos (Makkar, 2003).

Henke et al. (2016) encontraron que la adición de 1.5\% o más de TC de quebracho en la dieta de vacas lecheras afectó negativamente la digestibilidad de los diversos componentes de la misma. El consumo de taninos en cantidades equivalentes al $2 \%$ de la dieta es suficiente para inducir efectos indeseables como una disminución en la digestibilidad de la proteína de la dieta (Beauchemin et al., 2007). Aunque existen 
reportes de otros autores (Krueger et al., 2010; Ebert et al., 2017), que adicionan entre 1 y $1.5 \%$ de tanicos a la dieta y no modifican el consumo voluntario ni la respuesta productiva en bovinos de engorda.

\section{CONCLUSIÓN}

La adición directa de taninos en las heces de los bovinos disminuye su producción de gas; sin embargo, cuando son incluidos como parte de la dieta ofrecida a los bovinos, carecen de impacto en la producción de gas de a partir de las heces de bovinos en engorda.

\section{AGRADECIMIENTOS}

Los autores agradecen a Ganadera Los Migueles, S.A. de C.V., a su Director Ing. Regulo Terraza Romero y al Gerente de Producción MVZ Billy Josué Cervantes Pacheco por las facilidades otorgadas en el uso de animales e instalaciones para la realización del presente trabajo.

\section{LITERATURA CITADA}

ADEOLA O. 1999. Nutrient management procedures to enhance environmental conditions: An introduction. Journal of Animal Science. 77:427-429. DOI: $10.2527 / 1999.772427 x$

ANALYTICAL SOFTWARE. Statistix User's Manual, Release 9.0. Analytical Software, Tallahassee, FL. 2007. http://dx.doi.org/10.1016/j.foodcont.2013.06.040

AOAC. Official methods of analysis. 1997. 16th ed. Arlington, VA, USA: Association of Official Analytical Chemists.

ARCHIBEQUE SL, Miller DN, Freetly HC, Ferrell CL. 2006. Feeding high-moisture corn instead of dry-rolled corn reduces odorous compound production in manure of finishing beef cattle. Journal of Animal Science. 84:1767-1777. DOI: 10.2527/jas.2005-448

BAE HD, McAllister TA, Yanke J, Cheng KJ, Muir AD. 1993. Effects of condensed tannins on endoglucanase activity and filter paper digestion by Fibrobacter succinogenes S85. Applied and Environmental Microbiolgy. 59:2132-2138. DOI: 0099-2240/93/072132-07

BARROS-RODRÍGUEZ M, Oña-Rodríguez J, Mera-Andrade R, Artieda-Rojas J, CurayQuispe S, Avilés-Esquivel D, Solorio-Sánchez J, Guishca-Cunuhay C. 2017. Degradación ruminal de dietas a base de biomasa pos-cosecha de Amaranthus cruentus: efecto sobre los protozoos del rumen y producción de gas in vitro. Revista de Investigaciones Veterinarias del Perú. 28:812-821. DOI: 10.15381/rivep.v28i4.13931

BEAUCHEMIN RA, McGinn SM, Martinez TF, McAllister TA. 2007. Use of condensed tannins extract from quebracho trees to reduce methane emission from cattle. Journal Animal Science. 85:1990-1996. DOI: 10.2527/jas.2006-686 
BERNAL L, Ávila P, Ramírez G, Lascano CE, Tiemann T, Hess H. 2008. Degradación de nutrientes y emisión de gases al fermentar ensilaje y heno de Calliandra calothyrsus y Vigna unguiculata en el sistema Rusitec. Asociación Latinoamericana de Producción Animal. 16:199-204. ISSN: 1022-1301.

BESERRA LM, Leal CM, Maia S, Fernades AL, Freitas LT. 2011. Plantas taaniniferas e o controle de nematoides gastrintestinais de pequenos ruminantes. Ciencia Rural. 41:1967-1974. ISSN: 0103-8478.

BRAKE DW, Titgemeyer EC, Bailey EA, Anderson DE. 2014. Small intestinal digestion of raw cornstarch in cattle consuming a soybean hull-based diet is improved by duodenal casein infusion. Journal Animal Science. 92:4047-4056. DOI: 10.2527/jas/2014-7908.

CÁRDENAS PA. 2012. Efectos de los taninos encontrados en las leguminosas tropicales utilizadas en la nutrición de rumiantes. Revista PECUS Colombia. (3):33-39. ISSN: 21458308.

CARDONA-IGLESIAS JL, Mahecha-Ledesma L, Angulo-Arizala J. 2016. Arbustivas forrajeras y ácidos grasos: estrategias para disminuir la producción de metano entérico en bovinos. Agronomía Mesoamericana. 28:273-288. ISSN: 1021-7444.

CARMONA JC, Bolívar DM, Giraldo LA. 2005. El gas metano en la producción ganadera y alternativas para medir sus emisiones y aminorar su impacto a nivel ambiental y productivo. Revista Colombiana de Ciencias Pecuarias. 18:49-63. ISSN: 0120-0690.

DHANOA MS, France J, Crompton LA, Mauricio RM, Kebreab E, Mills JAN, Sanderson R, Dijkstra J, López S. 2004. Technical note: A proposed method to determine the extent of degradation of a feed in the rumen from the degradation profile obtained with the in vitro gas production technique using feces as the inoculum. Journal Animal Science. 82:733-746. DOI: 10.1093/ansci/82.3.733

DUVAL A, Averous L. 2016. Characterization and physicochemical properties of condensed tannins from Acacia catechu. Journal of Agricultural and Food Chemistry. 64:1751-1760. DOI: 10.1021/acs.jafc.5b05671

EBERT PJ, Bailey EA, Shreck AL, Jennings JS, Cole NA. 2017. Effect of condensed tannin extract supplementation on growth performance, nitrogen balance, gas emissions, and energetic losses of beef steers. Journal Animal Science. 95:1345-1355. DOI: $10.2527 /$ jas.2016.0341

FASS. Guide for the Care and Use of Agricultural Animals in Research and Teaching. 2010. 3th ed. Champaign, IL, USA: Federation of Animal Science Societies. ISBN 975-1831706-11-0 https://www.asas.org/ag_guide_3rded/HTML5/index.html 
FRUTOS P, Hervás G, Giráldez FJ, Mantecón AR. 2004. Tannins and ruminant nutrition. Spanish Journal of Agricultural Research. 2:191-202. http://revistas.inia.es/index.php/sjar/article/view/73/0

GILROYED BH, Li C, Reuter T, Bauchemin KA, Hao X, McAllister TA. 2015. Influence of distiller's grains and condensed tannins in the diet of feedlot cattle on biohydrogen production from cattle manure. International Journal of Hydrogen Energy. 40:6050-6058. DOI: 10.1016/j.ijhydene.2013.07.014

GRAINGER C, BEAUCHEMIN KA. 2011. Can enteric methane emissions from ruminants be lowered without lowering their production?. Animal Feed Science and Technology. 166:308-320. DOI: 10.1016/j.anifeedsci.2011.04.021

HENKE A, Dickhoefer U, Westreicher-Kristen E, Knappstein K, Molkentin J, Hasler M, Susenbeth A. 2016. Effect of dietary Quebracho tannin extract on feed intake, digestibility, excretion of urinary purine derivatives and milk production in dairy cows. Archives of Animal Nutrition. 71:37-53. DOI: 10.1080/1745039X.2016.1250541

HICKS CR. 1973. Fundamental Concepts in the Design of Experiments. Holt, Reinhardt and Wiston, New York. USA. ISBN 003080132x.

HUANG XD, Liang JB, Tan HY, Yahya R, Ho YW. 2011. Effects of Leucaena condensed tannins of differing molecular weights on in vitro $\mathrm{CH} 4$ production. Animal Feed Science and Technology. 166:373-376. DOI: 10.1016/j.anifeedsci.2011.04.026

HUNTINGTON GB, Harmon DL, Richards CJ. 2006. Sites, rates, and limits of starch digestion and glucose metabolism in growing cattle. Journal Animal Science. 84 (Suppl 1):E14-E24. DOI: 10.2527/2006.8413_supplE14x

JONES GA, McAllister TA, Muir AD, Cheng KJ. 1994. Effects of sainfoin (Onobrychis viciifolia Scop.) condensed tannins on growth and proteolysis by four strains of ruminal bacteria. Applied and Environmental Microbiology. 60:1374-1378. DOI: 0099-2240/94

KARDEL M, Taube F, Schultz H, Gierus M. 2013. Different approaches to evaluate tannin content and structure of selected plants extracts - review and new aspects. Journal of Applied Botany and Food Quality. 86:154-166. DOI: 10.5073/JABFQ.2013.086.021

KEBREAB EJ, Dijkstra A, Bannink, France J. 2009. Recent advances in modeling nutrient utilization in ruminants. Journal Animal Science. 87(Suppl. 1), E111-E122. DOI: 10.2527/jas.2008-1313

KRUEGER WK, Gutierrez-Bañuelos H, Carstens GE, Min BR, Pinchak WE, Gomez RR, Anderson RC, Krueger NA, Forbes TDA. 2010. Effects of dietary tannin source on performance, feed efficiency, ruminal fermentation, and carcass and non-carcass traits in 
steers fed a high-grain diet. Animal Feed Science and Technology. 159:1-9. DOI: 10.1016/j.anifeedsci.2010.05.003

LASCANO C, Cárdenas E. 2010. Alternatives for methane emission mitigation in livestock systems. Revista Brasileira de Zootecnia. 39:175-182. DOI: 10.1590/S151635982010001300020

MAKKAR HPS. 2003. Effects and fate of tannins in ruminant animals, adaptation to tannins, and strategies to overcome detrimental effects of feeding tannin-rich feeds. Small Ruminant Research. 49:241-256. DOI: 10.1016/S0921-4488(03)00142-1

MILLER DN, Berry ED, Wells JE, Ferrell CL, Archibeque SL, Freetly HC. 2006. Influence of genotype and diet on steer performance, manure odor, and carriage of pathogenic and other fecal bacteria. III. Odorous compound production. Journal Animal Science. 84:25332545. DOI: 10.2527/jas.2005-748

MILLER DN, Varel VH. 2001. In vitro study of the biochemical origin and production limits of odorous compounds in cattle feedlots. Journal Animal Science. 79:2949-2956. DOI: 10.2527/2001.79122949x

NASEM. The National Academies of Science, Engineering, and Medicine. 2016. Nutrient Requirements of Beef Cattle. National Academy Press. 8th ed. Washington, DC, USA.

OWENS FN, Zinn RA, Kim YK. 1986. Limits to starch digestion in the ruminants small intestine. Journal Animal Science. 63:1634-1648. DOI: 10.2527/jas.1986.6351634x

SHABTAY A, Ravid U, Brosh A, Baybikov R, Eitam H, Laor Y. 2009. Dynamics of offensive gas-phase odorants in fresh and aged feces throughout the development of beef cattle. Journal Animal Science. 87:1835-1848. DOI: 10.2527/jas.2008-1357

VAREL VH, Miller DN. 2000. Plant-derived oils reduce pathogens and gaseous emissions from stored cattle waste. American Society for Microbiology. 3:1366-1370. DOI: 10.1128/AEM.67.3.1366-1370.2001

VÁZQUEZ AA, Álvarez E, López JA, Wall A, De la rosa LA. 2012. Taninos hidrolizables y condensados: naturaleza química, ventajas y desventajas de su consumo. Tecnociencia Chihuahua. 6:84-93.

http://tecnociencia.uach.mx/numeros/v6n2/data/Taninos_hidrolizables_y_condensados_ naturaleza_quimica_ventajas_y_desventajas_de_su_consumo.pdf

VÉLEZ-TERRANOVA M, Campos-Gaona R, Sánchez-Guerrero H. 2014. Uso de metabolitos secundarios de las plantas para reducir la metanogénesis ruminal. Tropical and Subtropical Agroecosystems. 17:489-499. E-ISSN: 1870-0462 